Portland State University

PDXScholar

\title{
Right-Hook Crash Scenario: Effects of Environmental Factors on Driver's Visual Attention and Crash Risk
}

\author{
Mafruhatul Jannat \\ Oregon State University \\ Hagai Tapiro \\ Ben-Gurion University of the Negev \\ Christopher Monsere \\ Portland State University, monsere@pdx.edu \\ David Hurwitz \\ Oregon State University
}

Follow this and additional works at: https://pdxscholar.library.pdx.edu/cengin_fac

Part of the Transportation Engineering Commons

Let us know how access to this document benefits you.

\section{Citation Details}

Jannat, M., Tapiro, H., Monsere, C., \& Hurwitz, D. S. (2020). Right-Hook Crash Scenario: Effects of Environmental Factors on Driver's Visual Attention and Crash Risk. Journal of Transportation Engineering, Part A: Systems, 146(5), 04020026.

This Post-Print is brought to you for free and open access. It has been accepted for inclusion in Civil and Environmental Engineering Faculty Publications and Presentations by an authorized administrator of PDXScholar. Please contact us if we can make this document more accessible: pdxscholar@pdx.edu. 


\title{
Right-Hook Crash Scenario: Effects of Environmental Factors on Driver's Visual Attention and Crash-Risk
}

\author{
Mafruhatul Jannat ${ }^{\mathrm{a}}$, Hagai Tapiro ${ }^{\mathrm{b}}$, Chris Monsere ${ }^{\mathrm{c}}$, and David S. Hurwitz ${ }^{\mathrm{d}}$ \\ ${ }^{a}$ Saxton Lab Manager/Transportation Research Engineer, Leidos, medha.jannat@gmail.com \\ ${ }^{\mathrm{b}}$ Post-Doc, School of Civil and Construction Engineering, Oregon State University, \\ hagai.tapiro@oregonstate.edu \\ 'Professor and Chair, Department of Civil and Environmental Engineering, Portland State \\ University, monsere@pdx.edu \\ ${ }^{\mathrm{d}}$ Corresponding Author, Associate Professor, School of Civil and Construction Engineering, \\ Oregon State University, david.hurwitz@oregonstate.edu
}

\section{ABSTRACT}

A right-hook $(\mathrm{RH})$ crash is a common type of bicycle-motor vehicle crash that occurs between a right-turning vehicle and through-moving bicycle at an intersection in right hand driving countries. Despite the frequency and severity of this crash type, no significant driver-

performance based evidence of the causes of RH crashes at signalized intersections was found in the literature. This study examined the driver's visual attention in a right-turning scenario at signalized intersections with bicycle lanes but no exclusive right-turning lanes while interacting with a bicyclist to develop an understanding of RH crash causality. Fifty-one participants in 21 simulated road scenarios performed a right-turning maneuver at a signalized intersection while conflicting with traffic, pedestrians and bicyclists. Overall, a total of $820(41 * 20)$ observable right-turn maneuvers with visual attention data were analyzed. The results show that in the presence of conflicting oncoming left-turning vehicular traffic, drivers spent less visual attention on the approaching bicyclist, thus, making them less likely to be detected by the driver. The presence of oncoming left-turning traffic and the, bicyclist's speed and relative position, and conflicting pedestrians were found to likely increase the risk of $\mathrm{RH}$ crashes. The results of the 
current study will help to identify effective crash mitigation strategies which may include improving the vehicle-human interface or the implementation of design treatments in the road environment to improve driver and bicyclist performance.

31 Keywords: Bicycle-motor vehicle Crash, Right hook crash, Bicyclist, Road safety, Driving

32 simulator, Driver behavior

\section{BACKGROUND}

34 Most bicycle-motor vehicle (BMV) crashes occur at intersections in urban areas-with crashes 35 involving right-turning vehicles and through moving bicycles, which are commonly termed as 36 "right-hook (RH) crashes" (see Fig. 1). According to the Oregon (OR) Bicycle Manual, "A RH

37 crash occurs when a right-turning driver crosses the path of a through bicyclist at an intersection" 38 ( Oregon Department of Transportation (ODOT) 2016). The United States National Highway 39 Traffic Safety Administration (NHTSA) categorized this crash type as "parallel path" crash 40 under "driver turn/merge into path of bicyclist" subgroup in NHTSA Manual Accident Typing 41 (MAT) for Bicyclist Accidents Coder's Handbook (Karsch et al. 2012; Hunter et al. 1995), when 42 the driver was making a right-turn and the bicyclist was riding in the same or opposite direction 43 of traffic. RH crashes at intersections can occur as the result of several scenarios of traffic 44 control and lane geometries at the intersection. This study examined the specific case of RH 45 crashes after the start-up period at a signalized intersection with no dedicated turning lane. In this scenario (sometimes referred to as "stale" green) both conflicting vehicles (the bicyclist and the

47 car) are moving. A RH crash in this condition can occur when a bicyclist overtakes a slow-

48 moving vehicle on the right and the vehicle unexpectedly makes a right-turn, or when a fastmoving vehicle overtakes the bicyclist and then tries to make a right-turn directly in front of the 50 bicyclist. 
NHTSA reports that there were 840 fatal bicycle-related crashes in 2016, which

52 accounted for $2.2 \%$ of transportation-related fatalities. NHTSA reported that $71 \%$ of fatal bicycle

53 crashes occurred in urban areas in 2016, with $30 \%$ of them at intersections. The literature

54 identifies intersections as hot-spots for bicycle-motor vehicle-related crashes (Korve and

55 Niemeier 2002; Wachtel et al. 1994; Wang and Nihan 2004; Weigand 2008).

56 To safely accomplish the dynamic and multifaceted driving task, drivers need to perceive,

57 identify, and correctly interpret the elements of the current traffic situation including

58 immediately adjacent traffic, road signs, route direction, and other inputs, while being vigilant

59 for obstacles and making predictions of near future traffic conditions to maintain control,

60 guidance, and navigation of the vehicle (Baumann et al. 2007).

61 Improper allocation of visual attention has been recognized for some time as a causal

62 factor in vehicular crashes (Treat et al. 1979). A NHTSA study confirmed that $55.7 \%$ of

63 intersection-related crashes occurred due to drivers' recognition errors such as inattention,

64 internal and external distractions, or inadequate surveillance (NHTSA 2010). The most

65 frequently assigned critical reason was found to be inadequate surveillance, which constituted

$6644.1 \%$ of all intersection-related crashes. Inadequate surveillance occurs when the driver is in a

67 situation where they need to scan a certain location to safely complete a maneuver and they

68 either fail to look in the appropriate place or looks but does not see. This failure can occur at an

69 intersection when the driver looks in the required direction before making a turn but fails to see

70 the approaching traffic (Dingus et al. 2006); or when the driver fails to identify the visual cue on

71 time, since the visual cue is in an unexpected location or incompatible with the driver's schemes

72 (Borowsky et al. 2008). 
Driver's visual attention was also found to be a factor in the case of motor-bicycle

74 crashes. One of the major contributing factors to this crash type is the improper allocation of

75 driver's visual attention while making turns at an intersection. Before making a right turn, drivers

76 focus their visual attention on the cars coming from the left and fail to detect the bicyclist

77 coming from their right early enough to respond safely, even when the bicyclist could be easily

78 detected (Summala et al. 1996; Wachtel et al. 1994). In the case of a bicyclist coming from an

79 unexpected direction, prior research found that even if drivers looked in the relevant direction

80 and noticed the bicyclist, often the identification was too late to effectively stop or yield

81 (Räsänen and Summala 1998).

It is worth noting that although the topic of right-turning vehicle crashes with bicycles appears in the literature with some frequency (Summala 1988; Wachtel et al. 1994; Weigand

84 2008), comparably little substantive research has been conducted on this topic. Improper

85 allocation of drivers' visual attention and inadequate surveillance methods were demonstrated as factors contributing to crashes between a driver turning right and a bicyclist from previous

87 studies. A safe right-turning maneuver requires that the driver will look and detect the bicyclist,

88 so their decision to make the right turn will be based on that information and corresponding conditions at the intersection. It was the goal of this study to measure the driver's visual attention

90 in these cases to identify the scenarios that increase the risk of a RH crash. The study hypothesis

91 was that right-turning driver's visual attention would be influenced by the relative position of

92 bicyclists and other visual cues in the driving environment; thereby bicyclists' relative position

93 and speed would increase the crash risk. The primary failure mechanism would be drivers who

94 fail to detect the bicyclist when approaching from behind in the driver's blind spot as compared 95 to when the bicyclist is riding in front of the driver in her focal vision. 


\section{RESEARCH METHODOLOGY}

\section{Participants}

A total of 67 individuals, primarily from the community surrounding Corvallis, OR, participated

99 in the driving simulator study. The responses recorded from 16 participants who exhibited

100 simulator sickness, were excluded from the original data set. As such, the results of 51

101 participants (30 males, 21 females) aged 19-69 (mean=30.24) were included in the analysis. All

102 participants had a valid driving license with at least one year driving experience and were

103 required to declare that they were still mentally and physically fit to drive at the time of the

104 experiment. Participants were given \$20 compensation in cash for participating in the

105 experiment.

106 Apparatus

107 The driving simulator

108 The Oregon State University (OSU) Driving Simulator is a high-fidelity, motion-based

109 simulator, consisting of a full 2009 Ford Fusion cab mounted above an electric pitch motion

110 system capable of rotating \pm 4 degrees (see Fig. 1. Schematic description of a right-hook crash.

111

112 Fig. 2). The vehicle cab is mounted on the pitch motion system with the driver's located at the

113 center of the viewing volume. The pitch motion system allows for the accurate representation of

114 acceleration or deceleration (Oregon State University 2011). Three projectors with a resolution

115 of 1,400 by 1,050 are used to project a front view of 180 degrees by 40 degrees on three adjacent

116 screens, measure 3.4 meters by 2.3 meters each. A digital light-processing projector is used to

117 display a rear image for the driver's center mirror and the two side mirrors have embedded liquid

118 crystal displays (LCD). The simulator is equipped with a surround sound system that produces 
119 ambient and driving sounds. The simulator software is capable of capturing and outputting

120 highly accurate values for performance measures such as speed, position, brake, and

121 acceleration. The virtual environment was developed using Simcreator simulator software

122 package by Realtime technologies (RTI), Internet Scene Assembler (ISA) and Google Sketchup.

123 Eye-tracking

124 Eye-tracking data were collected with the Mobile Eye-XG platform from Applied Science

125 Laboratories (ASL) as displayed in Fig. 3. This platform allows the user to have both

126 unconstrained eye and head movement. A sampling rate of $30 \mathrm{~Hz}$ was used, with an accuracy of

127 0.5-1.0 degrees (Oregon State University 2011). The participant's gaze was calculated based on

128 the correlation between the participant's pupil position and the reflection of three infrared lights

129 on the eyeball. The system records a fixation when the participant's eyes pause in a certain

130 position for more than 100 milliseconds.

131 Eye-Fixation Data Reduction

132 The eye fixation data analysis process was performed on 25 second video clips capturing each

133 participant's approach to an intersection preparing to and completing a right turn. Each video

134 clip started from the point when the participant approached the intersection and ended when the

135 participant completed the right-turn maneuver. The participant's eye movement data was

136 analyzed with ASL Results Plus software. For this process, researchers watched each collected

137 approach video (20 per participant) and drew AOI (area of interest) polygons on individual video

138 frames in a sequence separated by intervals of approximately 5-10 frames. Once the researcher

139 manually situated each AOI, the Results Plus software automatically identified the fixations

140 inside each AOI (i.e., traffic signals (overhead and post-mounted), pedestrians, bicyclists,

141 mirrors (rear and driver's right side), and oncoming left turning vehicles) (See Fig. 4). At the end 
142 of the process a data set was exported from the Results Plus software that summarizes the

143 fixations data during a single 25-second intersection approach video for further statistical

144 analysis. The data included: the number of fixations, total fixation durations (secs), average

145 fixation durations (secs), and time of the first fixation within each AOI created during an

146 intersection approach and right-turn maneuver. Fixations outside of coded AOIs were universally

147 defined as OUTSIDE and were not analyzed further.

\section{Driving scenarios}

149 Twenty-one different right-turning road scenarios within seven different driving tracks were

150 constructed in a typical suburban-like virtual environment where shops and housing buildings are

151 placed along the sides of the road. In each, a cross section of the roadway included three 3.6

152 meters traffic lanes with 1.7 meters bicycle lanes in each direction was presented. In the driver's

153 direction of travel, the intersection approach was a single shared through and turning lane. In the

154 opposing direction, there were two lanes. No exclusive left-turn or right-turn bay was provided at

155 the intersection. The receiving roadway for the right turn had a single receiving lane. The

156 intersection approaches had a posted speed limit of $15.65 \mathrm{~meter} / \mathrm{second}(\mathrm{m} / \mathrm{s})(35 \mathrm{miles} / \mathrm{hour}$

157 (mph)) (see Fig. 5). The scenarios introduced a combination of four independent variables

158 resulted in 20 right-turning scenarios that were presented to the participant. The scenarios

159 introduced a variation of on-coming traffic, crossing pedestrian, and traveling bicyclist's position

160 and speed (see study design). The movements of the other dynamic actors in the scene were

161 initiated with proximity sensors coded in the simulation in response to the position and speed of

162 the subject vehicle. The oncoming left-turning vehicles start their movement on the green light,

163 while the driver is waiting at the red light at the intersection. As the driver approached the

164 signalized intersection, the pedestrian entered the conflicting crosswalk to cross the road. The 
movement of the bicycle ahead was synced with the movement of the vehicle, so, when the driver was approaching the intersection the bicyclist ahead of him was also moving toward the intersection. The bicyclists from behind condition was designed in a way that they were visible in drivers' rear view or side mirror while the drivers were approaching the intersection. The simulated environment was designed in a way that drivers could not see the bicyclists pulling onto the bike lane from the adjacent lane behind them (though they would have passed other bicyclists in the tangent sections so were aware bicycles were present in the simulation).

The design and sequencing of the 20 scenarios was influenced by a need to minimize the occurrence of simulator sickness. Therefore, the experimental driving was divided into seven individual driving tracks of intersections and each included 2-4 right-turning scenarios. Each scenario was assigned a position on a grid based on the assignment of random number generation. The order of presentation of driving tracks 1 to 6 was partly counterbalanced (i.e. there were four possible sequences of presentation to the driving tracks) to minimize the practice effect on driver performance and made it more difficult for participants to predict when the simulation would stop. Each participant was randomly assigned to drive the tracks in one of those orders. To provide more variability in the sequence of right turning scenario presentations, the start and finish locations of these driving tracks were not consistent. Also, the scenarios were interrupted by through movements at intersections that were not experimental scenarios to prevent participants anticipating the motivation for the study and to reduce simulator sickness.

Participants were given the instruction to turn right at an intersection through an automated voice command saying: "Turn Right at the Next Intersection", 100 meters upstream of the intersection. This voice command was automatically generated on the vehicle approach to the intersection. Fig. 6 shows an example driving track layout of three right-turning scenarios (e.g., tracks 1, 2 
and 7). The "Path" in the figure indicates the sequence of maneuvers participants were asked to perform.

\section{Experimental procedure}

191 Upon arrival, the participant was presented with an informed consent document that provided a 192 general description of the entire experiment and the opportunity to ask clarifying questions.

193 Participants were informed that they could stop the experiment at any time for any reason and 194 still receive full compensation. Participants were not told of the specific research objective or the 195 associated hypotheses. Participants completed a prescreening demographic survey, including 196 questions related to: age, gender, driving experience, highest level of education, use of corrective

197 glasses or contact lenses, as well as their prior experience with both driving simulators and 198 motion sickness.

At this stage, participants were required to perform a 3- to 5-minute practice drive to 200 acclimate to the operational characteristics of the driving simulator, and to confirm if they 201 experienced simulator sickness at any point during the practice drive. Once seated in the vehicle, 202 participants were allowed to adjust the seat, rear-view mirror, and steering wheel to maximize 203 comfort and performance while driving in the experiment. Participants were also instructed to 204 drive and follow all traffic laws as they normally would. The calibration drive was conducted in 205 a generic city environment, as previously described, and drivers were required to make several 206 right turns. If a participant reported simulation sickness during or after the calibration drive, their 207 experimental work was stopped, they were fully compensated, and any recorded data was 208 excluded from further analysis.

Before starting the experimental drive, participants were instrumented with a headmounted eye tracker and performed a short calibration process. After that, participants received a 
211 brief instruction about the test environment and the tasks they were required to perform.

212 Participants were asked to perform right-turning maneuvers at signalized intersections. As noted

213 in the introduction, all participants approaching the intersection were presented a green signal

214 and were in motion. Participant's eye movements were collected while driving through 20

215 typical right-turning intersections in the simulated environment. As previously stated, the entire

216 experiment was divided into seven driving tracks that were presented in a random order and

217 random starting and ending points within each track. The virtual driving course took participants

21820 to 30 minutes to complete. The entire experiment, including the consent process, eye tracker

219 calibration and post-drive questionnaire, lasted approximately 50 minutes.

220 Study design

221 To measure participant's visual attention during the course of the right-turn maneuver, the

222 average total (summed) fixation duration (ATFD) was documented for each predefined dynamic

223 area of interest (AOI) in each scenario. Fig. 7 shows examples of different AOIs that drivers

224 fixated on during the experiment.

225 Analysis of fixations was conducted to investigate the percentage of drivers who fixated

226 on the bicyclist before turning right at the intersection. The determination of the fixation on a

227 bicyclist was limited to when a driver fixated directly on the bicyclist AOI. For example, a driver

228 who fixated on the rear view or side mirror, but did not fixate directly on the bicyclist coming

229 from behind and then turned-right without yielding to the bicyclist - these cases indicated that

230 driver failed to detect the bicyclist and were coded as "not fixated" in the analysis.

231 Independent variables 
232 The relative position and speed of bicyclist, presence of oncoming left-turning vehicular traffic,

233 and conflicting pedestrian in the crosswalk may influence drivers' visual attention while turning

234 right. Therefore, all these factors were included as independent variables.

235 The first independent variable "relative position of bicyclist" had three levels - 1) no

236 bicyclist, 2) bicyclist approaching from behind the driver, and 3) bicyclist riding ahead of the

237 driver. The second independent variable, bicyclist's speed had two levels -1$)$ lower $(5.36 \mathrm{~m} / \mathrm{s}$

$238(12 \mathrm{mph}))$, and 2) high $(7.15 \mathrm{~m} / \mathrm{s}(16 \mathrm{mph}))$. The third independent variable was the "presence of 239 oncoming left-turning vehicular traffic", which had two levels - 1) no oncoming (zero) vehicles 240 and 2) three oncoming vehicles. The last independent variable was the "presence of a conflicting

241 pedestrian in the crosswalk, which also had two levels - 1) no (zero) pedestrian and 2) one

242 conflicting pedestrian walking towards the participant.

243 Research Hypotheses

244 One of the common features of BMV crashes at intersections includes drivers' learned routine of 245 failing to account for an adjacent bicyclist before turning (Räsänen \& Summala, 1998). It was 246 hypothesized that right-turning driver's visual search would be influenced by the relative 247 position of bicyclists. It was inferred that the driver would fail to detect the bicyclist when 248 approaching from behind in the driver's blind spot as compared to when the bicyclist is riding in 249 front of the driver in his/her/their focal vision. Two hypotheses were formulated to address this:

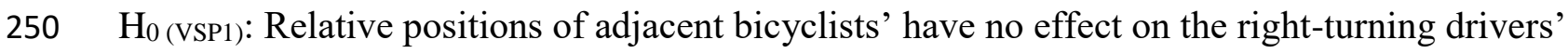
251 mean total fixation duration on areas of interest in the driving environment.

$252 \mathrm{H}_{0}$ (VSP2): There is no difference in the proportion of drivers who fixate on an adjacent bicyclist 253 during the right-turn maneuver at signalized intersections as the relative position of the bicyclist 254 changes. 
255 It has also been suggested that before turning right, drivers tend to focus their attention on the

256 cars coming from the left and fail to notice bicycles coming from their right early enough to

257 respond safely (Summala, Pasanen, Räsänen, \& Sievänen, 1996). Therefore, it was hypothesized

258 that driver's visual attention will be influenced when an oncoming car turns left in front of the

259 driver. Also, a study on bike boxes in Portland, Oregon suggested that the speed of bicyclists

260 overtaking the right-turning vehicle was a contributing factor to the occurrence RH crash (Dill,

261 Monsere, \& McNeil, 2012). It was inferred that bicyclist's speed would have an effect on the

262 visual attention of drivers while turning right during the latter portion of the green phase. Again,

263 the Institute of Transportation Engineers (ITE) Transportation Planning Handbook states that one

264 of the most common pedestrian crashes is the vehicle turn/merge conflict type (Meyer, 2009).

265 This conflict type occurs when a pedestrian and vehicle collide while the vehicle is conducting,

266 preparing, or has just completed a turning movement (Hurwitz \& Monsere, 2013). Considering

267 this finding, it was also hypothesized that the presence of a pedestrian in the conflicting

268 crosswalk might influence the visual attention of a right-turning driver.

$269 \mathrm{H}_{0}$ (VSP3): The speed of adjacent bicyclists have no effect on right-turning drivers' mean total

270 fixation duration on areas of interest in the driving environment.

$271 \mathrm{H}_{0}$ (VSP4): The presence of oncoming left-turning vehicular traffic has no effect on the right-

272 turning drivers' mean total fixation duration on areas of interest in the driving environment.

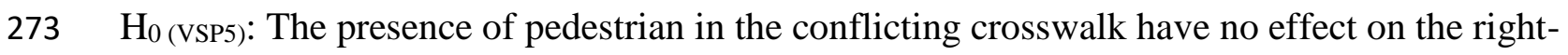

274 turning drivers' mean total fixation duration on areas of interest in the driving environment.

\section{Data Analysis}


276 Fifty-one participants successfully completed the driving simulator experiment. However, due to

277 eye-tracker calibration issues, completely usable data was only collected from 41 participants

278 representing a total of $820(41 * 20)$ observable right-turn maneuvers with visual attention data.

279 To test the five hypotheses stated above, for each of the four independent variables

280 (bicyclist's position, bicyclist's speed, oncoming vehicle presence, and pedestrian's presence) an

281 analysis of variance test (ANOVA) was conducted to statistically determine if there was any

282 difference in the ATFDs. However, when the variances were not equal (determined by Levene's

283 test) indicating the violation of the assumption of homogeneity of variance, the Welch's Robust

284 test or Omnibus F were used to interpret the F-statistic. Finally, pairwise comparisons were

285 calculated with Tukey’s Honest Significant Difference (HSD) test.

\section{RESULTS}

Forty-one participants (in total of 820 observable right-turn maneuvers with visual

289 attention data). To detect crashes, the driving task in the simulated environment was observed

290 continuously from the simulator's operator station and records were taken at the moment a crash

291 occurred. Drivers were also asked at the end of the experiment if they were involved in any

292 crashes during the experiment. The recorded crash data was further validated by checking the

293 locations of the subject vehicle and bicycle centroid, recorded as a dynamic variable data in the

294 driving simulator. In most cases, drivers could not notice when a crash occurred due to their

295 inadequate surveillance behavior and overloaded working memory during turning maneuver. A

296 Chi-square test was conducted for each of the independent variables to reveal significant

297 differences in the risk of a crash.

Fig. 8 shows the ATFD values and 95\% CIs for four AOIs at an intersection scenario

299 where the driver was presented with no pedestrians, no oncoming vehicles, and no bicyclists. 
This particular intersection is the most basic of all intersections shown to the participants. This scenario presented the simplest driving scenario to the driver.

Fig. 9 shows the ATFDs from all participants at an intersection where the bicyclist was approaching from behind the driver at $7.15 \mathrm{~m} / \mathrm{s}$, oncoming vehicles were present, and a pedestrian was present in the conflicting crosswalk. This case includes the greatest number of experimental variables, and is one of the most visually complex scenario.

\section{Bicyclist's relative position}

Three possible conditions existed for the bicyclist's position, the bicyclist was either riding ahead of the driver, approaching from behind the driver, or there was no bicyclist. The first two conditions were included in eight experimental scenarios each and the third level (no bicyclist) resulted in four experimental scenarios. The dataset was aggregated this way to isolate the impact of individual variable levels. Fig. 10 shows boxplots of ATFDs on each AOI for the bicyclist conditions. The boxplots display the distribution of ATFD in quartiles and indicat the mean and median of those distributions. The results of the ANOVA and pairwise comparisons presented in Table 1 shows that ATFDs on the bicyclist, pedestrian, right-side mirror, and oncoming vehicles had statistically significant differences. A two-sided Welch's two sample ttest indicated a statistically significant difference in ATFDs on bicyclists with respect to bicyclists' position. Drivers spent more time fixating on bicyclists when they were riding ahead as compared to when bicyclists were approaching from behind. The ATFD for the pedestrian AOIs was different when the bicyclist was riding in front vs when the bicyclist was approaching from behind with statistical significance. This finding revealed that in the presence of a bicyclist in the forward field of view, drivers spent less time fixating on the pedestrian compared to when the bicyclist was approaching from the behind. Similar findings were observed in the case of the 
323 oncoming vehicle AOI. However, a statistically significant difference in the ATFDs on the right-

324 side mirror and corresponding pairwise comparison showed that drivers spent more time fixation

325 on the right-side mirror when a bicyclist was approaching from behind compared to when there

326 was no bicyclist present at the intersection. No other significant differences were found with

$32795 \%$ confidence.

328 Thirteen crashes occurred when the bicyclist approached from behind and in the

329 remaining two crash incidents the bicyclist was riding ahead of the driver. A Chi-square test

330 revealed a statistically significant difference between these two bicyclist positions $(\mathrm{p}<0.01)$ with

331 respect to the occurrence of a crash.

332 Detecting the bicyclist

333 As summarized in Table 2 there were 328 (41 participants*8 turns) right-turns scenarios for each

334 bicyclist position. When the bicyclist was riding ahead of the driver in the forward field of view,

335 in $87 \%$ of the cases the drivers fixated on the bicyclist, i.e. actively scanned for the bicyclist

336 before turning right. However, when a bicyclist was approaching from behind, in only $44 \%$ of

337 the scenarios did a driver fixate on the bicyclist before turning right. A Chi-square test revealed a

338 statistically significant difference ( $\mathrm{p}$-value $<0.001)$ between the frequencies of driver fixation on

339 the bicyclist with different bicyclist positions.

340 Speed of Approaching Bicyclist

341 A comparison of ATFDs with respect to the bicyclist's speed was also conducted. Bicyclists

342 traveled at either $7.15 \mathrm{~m} / \mathrm{s}$ or $5.36 \mathrm{~m} / \mathrm{s}$. These two conditions consisted of eight experimental

343 scenarios each. The boxplot of ATFDs on AOIs by bicyclists speed is presented in Fig. 11.

344 Table 3 presents the results of two-sample, two-sided t-tests that were conducted to

345 determine the difference in the ATFDs with respect to bicyclist's speed. A statistically 
346 significant difference was found only in the ATFDs on the rear-view mirror with changes in the

347 bicyclist's speed. When bicyclist's speed was lower $(5.36 \mathrm{~m} / \mathrm{s})$, drivers spent more time scanning

348 the rear-view mirror compared to higher $(7.15 \mathrm{~m} / \mathrm{s})$ speed scenarios. This was likely because the

349 bicyclist required more time to travel the same distance before reaching the intersection in the

350 lower speed condition compared to the higher speed condition, while the driver yielded for the

351 bicyclist to pass.

In 12 out of the 15 crashes occurred when the bicyclist approached at $7.15 \mathrm{~m} / \mathrm{s}$ speed and

353 in the remaining three crashes had bicyclists approaching at $5.36 \mathrm{~m} / \mathrm{s} \mathrm{speed}$. A Chi-square test

354 revealed a statistically significant difference between bicyclist speeds ( $\mathrm{p}$-value $<0.05)$.

\section{Presence of oncoming left turning vehicle}

356 There were two levels of oncoming left turn vehicular traffic in the experiment (No vehicles and

3573 vehicles). These two conditions consisted of 10 experimental scenarios each. Fig. 12 shows the

358 boxplot of ATFDs on AOIs by the presence of oncoming left turn vehicular traffic. Table 4

359 presents the results of two-sample, two-sided t-tests that were conducted to determine the

360 difference in the ATFDs with respect to presence of oncoming vehicle. Statistically significant

361 differences indicated that drivers spent less time fixating on pedestrians, bicyclists riding ahead

362 of the driver, and the side signal when there were oncoming left-turn vehicles as compared

363 to when there was no oncoming left-turn vehicle present.

364 Eight crashes occurred when oncoming left-turning vehicles were present, and seven

365 crashes occurred when no oncoming vehicle was present. No statistically significant difference

366 was found for the presence of oncoming vehicles with respect to crash outcome.

\section{Presence of pedestrian}


368 Ten experimental scenarios presented a single pedestrian in the crosswalk and ten experimental 369 scenarios had no pedestrian present on the crosswalk. Fig. 13 shows the boxplot of ATFDs on 370 AOIs by the presence of a conflicting pedestrian.

From the result of two-sample, two-sided Students or Welch's t-tests, the only statistical

372 significant different in ATFD was found in the bicyclist behind AOI with the presence of a

373 pedestrian (Table 5). Results indicated that drivers spent more time fixating on the bicyclist

374 approaching from behind when a conflicting pedestrian was present in the crosswalk as

375 compared to when no pedestrian present. No statistically significant difference was found for the 376 presence of pedestrian with respect to crash outcomes.

\section{DISCUSSION}

378 This study investigated driver's visual attention and the risk of crash in a simulated virtual 379 environment while performing a right turn at a signalized intersection when a bicyclist is present 380 and in different circumstances (i.e. a pedestrian in the conflicting crosswalk and oncoming left turn vehicles) that might affect the driver's visual attention. The aim of this study was to identify

382 scenarios in the driver's visual search that increase the risk of a RH crash with the bicyclist. The 383 ATFD within a prescribed AOI was used to measure driver's visual attention on different targets. 384 Findings related to each research question on driver's visual attention are summarized below.

Aligned with the study hypothesis, a statistically significant difference (p-value $<0.001$ ) was found in the ATFDs on adjacent bicyclist between when a bicyclist was approaching from

387 behind and when a bicyclist was riding ahead of the driver. This circumstance also increases the 388 crash risk. This finding is consistent with the finding of Falzetta (Falzetta, M. (2004). A 389 Comparison of driving performance for individuals with and without Attention-DeficitHyperactivity Disorder. Unpublished Masters Thesis, Clemson University, Psychology 
391 Department, Clemson, SC.), where it was found that participants detected forward events more

392 successfully than rear events, and the location effect was consistent with an attention allocation

393 strategy that gave higher priority to the road ahead. A statistically significant difference (p-value

$394<0.001)$ was observed between the frequencies of driver fixations on the bicyclist when the

395 bicyclist was approaching from behind (44\%) vs. when bicyclist was riding ahead (87\%). Such

396 scanning behavior places bicyclists approaching from behind in a more vulnerable situation

397 where they are not detected by a driver at an intersection, contributing to the occurrence of $\mathrm{RH}$

398 crashes.

399 Statistically significant differences were also observed in the visual attention allocated to

400 conflicting pedestrians and oncoming left turn vehicles with respect to bicyclist's position. This

401 finding might suggest that when a bicyclist was riding ahead in the driver's visual field, drivers

402 anticipated a potential risk of collision with them more so than when they were approaching

403 from behind. However, when the bicyclist was approaching from behind, drivers spent more time

404 fixating on other traffic elements immediately relevant to the safe operation of the vehicle.

405 Another statistically significant finding was observed in the ATFDs on the right-side mirror

406 when the bicyclist was approaching from the behind compared to when there was no bicyclist.

407 This suggests that when drivers detected a bicyclist approaching from behind in the right-side

408 mirror, they spent more time fixating on the right-side mirror while waiting for the bicyclist to

409 pass through the intersection compared to when there was no bicyclist present. Bicyclist's speed

410 when approaching from behind had a statistically significant effect only on the visual attention

411 allocated to the rear-view mirror. A bicyclist that was detected in the rear-view mirror would

412 require more time to travel the same distance before reaching the intersection at the lower speed. 
413 Therefore, it can be assumed that the total fixation duration on checking the rear-view mirror in 414 search of the bicyclist was higher when the bicyclist traveled at a lower speed.

Oncoming left-turning traffic had a meaningful effect on the driver's visual attention

416 spread, demonstrated in the ATFDs on the side traffic signal, crossing pedestrian, and a bicyclist

417 riding ahead. Results suggest that in the presence of oncoming traffic, drivers spent less time

418 checking on other traffic elements in their focal vision, such as scanning for the pedestrian,

419 checking for the traffic signal status, or fixating on the bicyclist ahead. In the presence of

420 oncoming vehicular traffic, drivers spent a significant part of their time fixating on the oncoming

421 traffic, to the expense of the other traffic elements. The preferential visual attention oncoming

422 traffic gets from the driver over other road users and elements was observed in other

423 circumstances. In previous laboratory experiment it was observed that drivers' visual attention

424 was drawn to the oncoming traffic on the expense of pedestrians (Hurwitz \& Monsere, 2013),

425 and left turning drivers at signalized intersections were less likely to seek out for additional cues

426 from the road environment in the presence of opposing traffic (Knodler and Noyce 2005). In the

427 analysis of bicycle-car collisions at non-signalized intersections in the Helsinki City area,

428 Finland, by assessing the visual scanning behavior of drivers, researchers had found that drivers

429 develop a visual scanning strategy which concentrates on detection of more frequent and major

430 dangers, such as conflicting vehicles but ignores and may even mask visual information on less

431 frequent dangers, such as bicyclists (Summala et al. 1996). The driver possesses only a limited

432 capacity for visual attention, and so in accordance with the results from the current and previous

433 studies (Hurwitz S and Monsere 2013; Knodler and Noyce 2005; Summala et al. 1996) the

434 presence of oncoming vehicles perceived by the driver as posing more of a collision risk as 
compared to other objects in the road environment (like the bicyclists), and as a result of that the driver consistently spends more time fixating on the oncoming vehicles.

The presence of pedestrians also affected the driver's visual attention to the bicyclist approaching behind him, yet not the risk of a crash. Results were suggestive that when drivers were waiting for the conflicting pedestrian to pass through the intersection, they spent more time on fixating on the bicyclists approaching from behind compared to when there was no pedestrian, but not on the bicyclist that ride ahead from the driver. This was likely because while drivers were waiting for the pedestrian to pass through the intersection, they had more time to fixate on the bicyclist approaching from behind compared to when there was no crossing pedestrian.

Overall, this research provides valuable insights on the causal factors of RH crashes after the start-up period at a signalized intersection with no dedicated turning lane. These findings can help roadway engineers and planners while designing roadway sections and locations where bicycles are likely to be routinely overtaking motor vehicles on the right, especially at higher speeds. This can occur either in congested vehicle traffic or when bicycles have the advantage of a downgrade, as found in earlier studies. Findings from this study emphasizes the need for other design considerations to reduce RH crashes, for example additional pavement markings or signs may increase driver awareness. Other designs, such bending out the bicycle lane at the intersection or separating the bicycle movement with a separate signal phase may be feasible options. In bicycle-lane markings or minor speed humps may be effective at slowing bicycle speeds if other solutions are not feasible. To some degree, interactions at closely spaced signalized intersections in urban areas can be managed with careful thought of the bicycle and vehicle progression in platoons from upstream signals. This could be accomplished with a 
458 leading bicycle interval at the upstream signal that allows the majority of the bicycle platoon to 459 arrive ahead of vehicles (Kothuri et al. 2018).

\section{CONCLUSIONS}

461 The results indicate that bicyclist approaching from behind the driver in the blind spot is the most

462 vulnerable situation for a right-turning driver to fail to detect the bicyclist, potentially leading to

463 a RH crash. The presence of oncoming left-turning traffic and pedestrian at the crosswalk are

464 likely to increase the risk of RH crash, as they draw the driver's visual attention away from other

465 objects (e.g. the bicyclist). Results also indicate that higher speed bicyclists are likely to

466 contribute to the risk of RH crash.

As with any driving simulator experiment, while the various driver performance metrics

468 are measured robustly, it is not yet clear how to map the magnitudes of the differences to

469 expected crash outcomes. More work is needed to connect visual attention metrics and crash

470 outcomes. Additional variables could be included in the experiment to determine their effects on

471 the occurrence of right-hook crashes, for example the conspicuity of bicyclist, and time of day.

472 The assumption of constant speed of the approaching bicyclist is also limiting; in reality some

473 people on bicycles would slow down to avoid a collision or near collision. A study that included

474 dynamic bicycle approach speeds would be an improvement. Finally, one of the fundamental

475 limitations of within-subject design is fatigue effects that can cause participant's performance to

476 decline over time during the experiment. To mitigate this a larger sample of shorter drives might

477 reduce the risk of fatigue effect and simulator sickness, the experiment could be conducted in

478 two trials on two different days. Finally, the design of the experiment could be modified with

479 navigation tasks or other workloads enhancements so that the driver workload is more representative of actual conditions. 


\section{DATA AVAILABILITY}

482 Some or all data, models, and code generated or used during the study are proprietary or confidential in nature and may only be provided with restrictions (e.g. anonymized data).

Specifically, driver's visual attention data (number of fixations and durations) for each scenario aggregated by area of interest is available.

\section{ACKNOWLEDGEMENT}

The material in this paper is based on with supported by the ODOT under Grant No. SPR 767.

Any opinions, findings, conclusions, or recommendations expressed in this material are those of

the author and do not necessarily reflect the views of the ODOT. Additionally, this study was

conducted with support from the Oregon State Center for Healthy Aging Research, Life

Registry. The authors would like to thank Graduate Research Assistant Hisham Jashami for his assistance in preparing Figures $10-13$.

\section{REFERENCES}

Baumann, M. R. K., Rösler, D., and Krems, J. F. (2007). "Situation Awareness and Secondary Task Performance While Driving." Engineering Psychology and Cognitive Ergonomics, D. Harris, ed., Springer Berlin Heidelberg, Berlin, Heidelberg, 256-263.

Borowsky, A., Shinar, D., and Parmet, Y. (2008). "Sign location, sign recognition, and driver expectancies." Transportation Research Part F: Traffic Psychology and Behaviour, Elsevier Ltd, 11(6), 459-465.

Dill, J., Monsere, C. M., \& McNeil, N. (2012). Evaluation of bike boxes at signalized intersections. Accident Analysis \& Prevention, 44(1), 126-134.

Dingus, T. A., Klauer, S. G., Neale, V. L., Petersen, A., Lee, S. E., Sudweeks, J. D., Perez, M. A., Hankey, J., Ramsey, D. J., Gupta, S., and others. (2006). The 100-car naturalistic driving study, Phase II-results of the 100-car field experiment. National Highway Traffic Safety Administration (NHTSA), Washington, DC.

Falzetta, M. (2004). A Comparison of Driving Performance for Individuals With and Without Attention-Deficit-Hyperactivity Disorder. Unpublished Master's Thesis, Clemson University, Psychology Department, Clemson SC.

Hunter, W. W., Pein, W. E., \& Stutts, J. C. (1995). Bicycle-motor vehicle crash types: the early 1990s. Transportation Research Record, (1502), 65-74.

Hurwitz S, D., and Monsere, C. (2013). Improved Pedestrian Safety at Signalized Intersections Operating the Flashing Yellow Arrow. Oregon Transportation Research and Education 

Consortium. Portland, OR.

Karsch, H. M., Hedlund, J. H., Tison, J., \& Leaf, W. A. (2012). Review of Studies on Pedestrian and Bicyclist Safety, 1991-2007 (No. DOT HS 811 614).

Knodler, M. A., and Noyce, D. A. (2005). "Tracking Driver Eye Movements at Permissive LeftTurns." Proc., Third International Driving Symposium on Human Factors in Driver Assessment, Training and Vehicle Design, 134-142.

Korve, M. J., and Niemeier, D. A. (2002). "Benefit-Cost Analysis of Added Bicycle Phase at Existing Signalized Intersection.” Journal of Transportation Engineering, 128(1), 40-48.

Kothuri, S., Kading, A., Schrope, A., White, K., Smaglik, E., Aguilar, C., Gil, W. (2018). Eliminating the Right Hook: Safer Intersections for Bikes. Project NITC-RR-897. Portland, OR: Transportation Research and Education Center (TREC).

Meyer, Michael D (2009). Transportation Planning Handbook. 3rd ed. Institute of Transportation Engineers.

NHTSA. Traffic Safety Facts 2010: A Compilation of Motor Vehicle Crash Data from the Fatality Analysis Reporting System and the General Estimates System. (No DOT HS 811 659). U.S. Department of Transportation. Retrieved May 30, 2013 from http://wwwnrd.nhtsa.dot.gov/Pubs/811659.pdf.

Oregon Department of Transportation (ODOT). (2016). Oregon Bicyclist Manual. Oregon Pedestrian and Bicycle Program, Oregon.

Oregon State University. (2011). "OSU Driving Simulator." <http://cce.oregonstate.edu/drivingand-bicycling-simulator> (Feb. 10, 2018).

Räsänen, M., and Summala, H. (1998). "Attention and expectation problems in bicycle-car collisions: An in-depth study.” Accident Analysis and Prevention, 30(5), 657-666.

Summala, H. (1988). "Risk control is not risk adjustment: The zero-risk theory of driver behaviour and its implications." Ergonomics, 31(4), 491-506.

Summala, H., Pasanen, E., Räsänen, M., and Sievänen, J. (1996). "Bicycle accidents and drivers' visual search at left and right turns." Accident Analysis \& Prevention, Elsevier, 28(2), 147153.

Treat, J. R., Tumbas, N. S., McDonald, S. T., Shinar, D., and Hume, R. D. (1979). Tri-level study of the causes of traffic accidents. executive summary. National Highway Traffic Safety Administration (NHTSA), Washington, DC.

Wachtel, A., Lewiston, D., and others. (1994). "Risk factors for bicycle-motor vehicle collisions at intersections." ITE Journal(Institute of Transportation Engineers), 64(9), 30-35.

Wang, Y., and Nihan, N. L. (2004). "Estimating the risk of collisions between bicycles and motor vehicles at signalized intersections." Accident Analysis and Prevention, 36(3), 313321.

Weigand, L. (2008). A Review of Literature: Intersection Treatments to Improve Bicycle Access and Safety. Oregon Transportation Research and Education Consortium. Portland, OR. 
552 Table 1. ANOVA analysis of difference in ATFDs by bicyclist position.

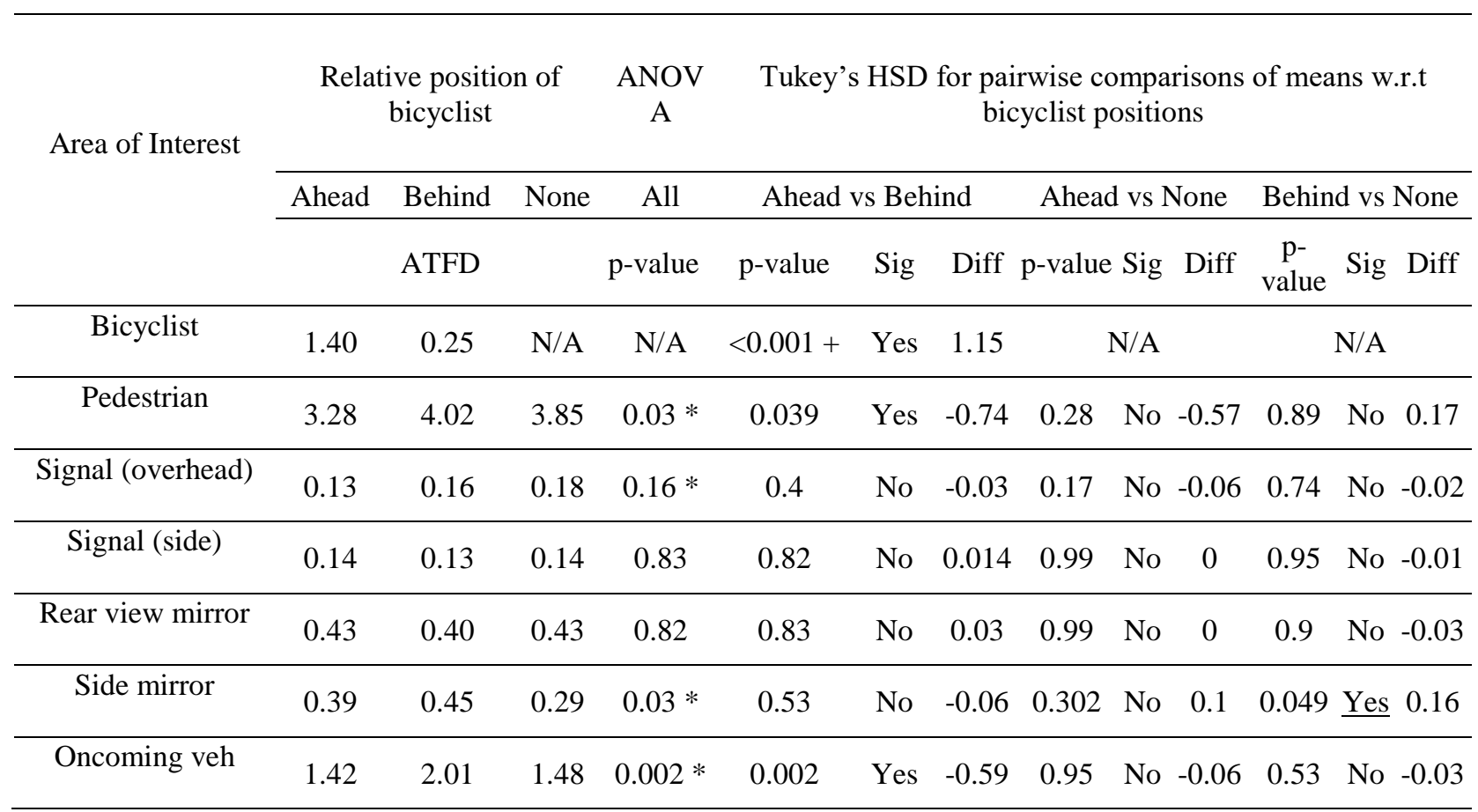

553 + No multiple comparisons required. P-value reflects a two-sided Welch's two sample t-test.

$554 *$ P-value reflects a Welch $\mathrm{F}$ test.

555

556 Table 2. Detecting of a bicyclist.

\begin{tabular}{ccc}
\hline \multirow{2}{*}{$\begin{array}{c}\text { Frequency } \\
\text { of fixation }\end{array}$} & \multicolumn{2}{c}{ Bicyclist position ${ }^{557}$} \\
\cline { 2 - 3 } & Ahead & Behind \\
\hline Total (n) & 328 & 328 \\
\hline Fixated & 284 & 145 \\
\hline$\%$ & $87 \%$ & $44 \%$ \\
\hline
\end{tabular}


Table 3. Two-sample $t$-test of ATFDs by bicyclist speed.

\begin{tabular}{ccccc}
\hline \multirow{2}{*}{ Areas of Interest } & \multicolumn{2}{c}{ Speed of Bicyclist } & \multicolumn{2}{c}{ Two sample two tail $t$-test } \\
\cline { 2 - 5 } & $7.15 \mathrm{~m} / \mathrm{s}$ & $5.36 \mathrm{~m} / \mathrm{s}$ & \multicolumn{2}{c}{$7.15 \mathrm{~m} / \mathrm{s}$ vs $5.36 \mathrm{~m} / \mathrm{s}$} \\
\cline { 2 - 5 } & \multicolumn{2}{c}{ ATFD $(\mathrm{sec})$} & p-value & Significant \\
\hline Pedestrian & 3.61 & 3.68 & 0.83 & No \\
\hline Bicyclist ahead & 1.43 & 1.38 & 0.78 & No \\
\hline Bicyclist behind & 0.20 & 0.30 & 0.98 & No \\
\hline Signal (overhead) & 0.14 & 0.14 & 1.00 & No \\
\hline Signal (side) & 0.14 & 0.13 & 0.91 & No \\
\hline Rear view mirror & 0.36 & 0.47 & $0.03+$ & Yes \\
\hline Side view mirror & 0.39 & 0.46 & $0.23+$ & No \\
\hline Oncoming veh & 1.89 & 1.54 & 0.06 & No/Suggestive \\
\hline
\end{tabular}

+ P-value reflects a two-sided Welch's two sample $t$-test

561 Table 4. Two-sample t-test of ATFDs comparing AOIs by oncoming left turn vehicles condition.

\begin{tabular}{ccccc}
\hline \multirow{2}{*}{ Areas of Interest } & \multicolumn{2}{c}{ Oncoming Vehicle } & \multicolumn{2}{c}{ Two sample two tail t-test } \\
\cline { 2 - 5 } & \multicolumn{2}{c}{3 Veh } & No Veh & \multicolumn{2}{c}{3 Veh vs No Veh } \\
\cline { 2 - 5 } & \multicolumn{2}{c}{ ATFD (sec) } & p-value & Significant \\
\hline Pedestrian & 3.11 & 4.26 & $<0.001+$ & Yes \\
\hline Bicyclist ahead & 1.20 & 1.61 & $0.01+$ & Yes \\
\hline Bicyclist behind & 0.21 & 0.29 & $0.09+$ & No \\
\hline Signal (overhead) & 0.16 & 0.14 & 0.57 & No \\
\hline Signal (Side) & 0.11 & 0.16 & $0.02+$ & Yes \\
\hline Rear view mirror & 0.38 & 0.46 & $0.11+$ & No \\
\hline Side view mirror & 0.39 & 0.40 & 0.87 & No \\
\hline Oncoming veh & 1.67 & N/A & N/A & N/A \\
\hline
\end{tabular}

+ P-value reflects a two-sided Welch's two sample t-test 
564 Table 5. Two-sample t-test of ATFDs comparing AOIs by Conflicting Pedestrian.

\begin{tabular}{ccccc}
\hline \multirow{2}{*}{ Areas of Interest } & \multicolumn{2}{c}{ Pedestrian } & \multicolumn{2}{c}{ Two sample two tail $t$-test } \\
\cline { 2 - 5 } & Ped & No Ped & \multicolumn{2}{c}{ Ped vs No Ped } \\
\cline { 2 - 5 } & ATFD $(\mathrm{sec})$ & & p-value & Significant \\
\hline Pedestrian & 3.69 & N/A & 0.88 & N/A \\
\hline Bicyclist Ahead & 1.39 & 1.42 & $<0.001+$ & No \\
\hline Bicyclist Behind & 0.38 & 0.12 & 0.35 & Yes \\
\hline Signal_Overhead & 0.14 & 0.16 & 0.72 & No \\
\hline Signal_Side & 0.17 & 0.10 & $0.06+$ & Suggestive \\
\hline RV_Mirror & 0.47 & 0.38 & 0.76 & No \\
\hline Side_Mirror & 0.40 & 0.39 & 0.99 & No \\
\hline Oncoming veh & 1.67 & 1.66 & & \\
\hline
\end{tabular}


566 Captions and Notes for Figures

567 Fig. 1. Schematic description of a right-hook crash.

568

569 Fig. 2. The OSU Driving Simulator from inside (a) and outside (b) the vehicle.

570

571 Fig. 3. OSU researcher demonstrating the Mobile Eye XG recording unit (image by David S.

572 Hurwitz).

573

574 Fig. 4. The ASL Results Plus software. In this frame the driver was fixating on a bicyclist before 575 turning right. This figure also includes heat maps (shaded circular patterns) for the conflicting 576 pedestrian AOI crossing the intersection and the side traffic signal AOI with green indication in 577 driver's field of view.

578

579 Fig. 5. Screen capture of intersection approach in the simulated environment, this scenario 580 includes the presence of oncoming left-turning vehicles waiting in the queue, and a bicyclist 581 riding ahead of the right-turning driver at the latter portion of green phase.

582

Fig. 6. Example driving track layout for tracks 1, 2 and 7 with three right-turning scenarios 584 path Start-Thru-Right-Thru-Right-Thru-Right-Finish.

585

586 Fig. 7. Examples of Different AOIs Drivers Fixated On During the Experiment 587

Fig. 8. ATFDs with 95\% CIs for Control Case (No Bicyclists, No Vehicles, No Pedestrians) 
590 Fig. 9. ATFD with 95\% CIs for One of the Most Visually Complex Scenario (Bicyclist

591 Approaching From Behind at $7.15 \mathrm{~m} / \mathrm{s}$, Three Vehicles, One Conflicting Pedestrian)

592

593 Fig. 10. Box plot of ATFDs at all intersections by bicyclist position.

594

595 Fig. 11. Box plot of ATFDs at all intersections, according to bicyclist's speed.

596

597 Fig. 12. Box plot of ATFDs at all intersections, according to the presence of oncoming left turn 598 vehicle.

599

600 Fig. 13. Box Plot of ATFDs at all Intersections by the Presence of Pedestrians. 